\title{
Total Thyroidectomy, Sternectomy and Rib Resection with Anterolateral Thigh Fasciocutaneous Flap for Advanced Follicular Thyroid Carcinoma \\ QY Ling ${ }^{1}$, S Nadarajan ${ }^{2}$
}

\begin{abstract}
Within this case report, I present a 59 years old male patient presented with a 10 year history of anterior neck and chest mass. FNAC showed the lesion is follicular thyroid neoplasm in origin. CT Neck and Thorax locally advanced malignancy of upper chest wall infiltrating the sternum and upper ribs. The patient was managed by multidisciplinary team (MDT) and subjected to total thyroidectomy, sternectomy and rib resection with Anterolateral Thigh Fasciocutaneous flap reconstruction. This case is a reminder that surgery is still the primary treatment of choice in these cases and subsequently followed by radioiodine ablation (RAI) with close monitoring of disease progression post-operatively. MDT involvement and surgery planning earlier proven to benefit patient especially in this case where Superior Thyroid Artery and Vein preserved by Endocrine Surgeons as the anastomosis recipient for Anterolateral Thigh Fasciocutaneous flap.
\end{abstract}

Keywords: Total Thyroidectomy, Metastatic Follicular Throid Carcinoma, Sternectomy, Rib Resection, Anterolateral Thigh Flap Reconstruction. Superior Thyroid Artery Anastomosis

From: ${ }^{1}$ Breast and Endocrine Unit, Department of Sugery, Universiti Kebangsaan Malaysia ${ }^{2}$ Vascular Unit, Department of Surgery, University Kebangsaan Malyasia.

Correspondence: Dr S Nadarajan, Department of Sugery, Hospital University Kebangsaan Malyasia, Jalan Yaacob Latif, 56000 Cheras, Malaysia. Fax: +603-91456684, e-mail: sivasurgery@gmail.com 


\section{INTRODUCTION}

Thyroid malignancies are the most common of all endocrine tumors with the follicular variant representing the second most common tumor subtype. Treatment is usually surgery with RAI to achieve total remission. Prognosis is excellent with a 10 year survival rate ia about $85 \%$. Dissemination of follicular thyroid carcinoma is primarily hematogenous which in advanced disease results in pulmonary and bone deposits. We report a case of follicular thyroid cancer with extensive mediastinal involvement and pulmonary deposits that required tumor extirpation, sternectomy and rib resection for palliation.

\section{CASE REPORT}

A 59 year old gentleman, with no prior medical illnesses, presented with a 10 year history of anterior neck swelling followed by anterior chest wall swelling for 8 years. However, 6 months prior to presentation the anterior chest wall mass enlarged rapidly and it became painful. He denies any airway symptoms but has minimal weight loss the past 6 months. He also complains of heat intolerance but no other thyroid hyperactivity symptoms. No voice change noted. Physical examination revealed a large $15 \times 10 \mathrm{~cm}$ anterior chest wall mass that is fixed to the skin and underlying structures. Overlying skin is normal with no color or consistency changes. The trachea remains central, however the external jugular veins were dilated. There is also a neck swelling measuring $5 \times 3 \mathrm{~cm}$, which is not fixed to the skin and moves with swallowing. Overlying skin remains normal. Biochemical parameters were within normal limits.

An initial computed tomography scan of the neck and thorax showed an enlarged right thyroid lobe with no retrosternal extension along with the presence of anterior mediastinal mass with local invasion to the sternum and ribs (Fig 1-6). Features are suggestive of 
malignant nature with multiple lung metastases from the anterior mediastinum mass. Fine needle aspiration biopsy of the neck mass was done and it showed features suggestive of a follicular lesion.

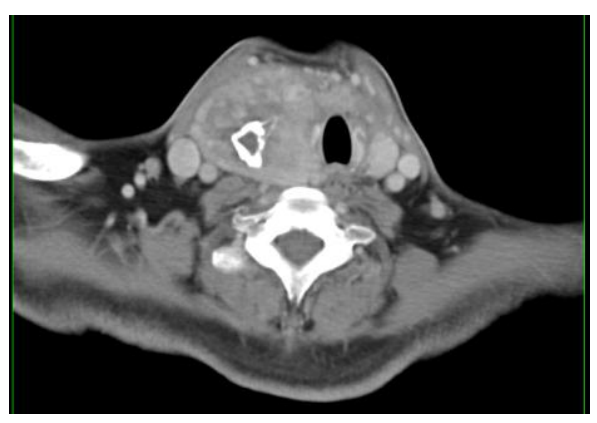

Fig 1

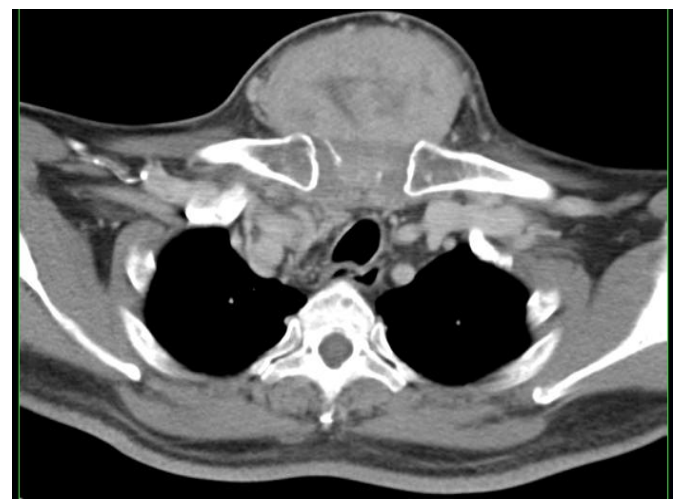

Fig 3

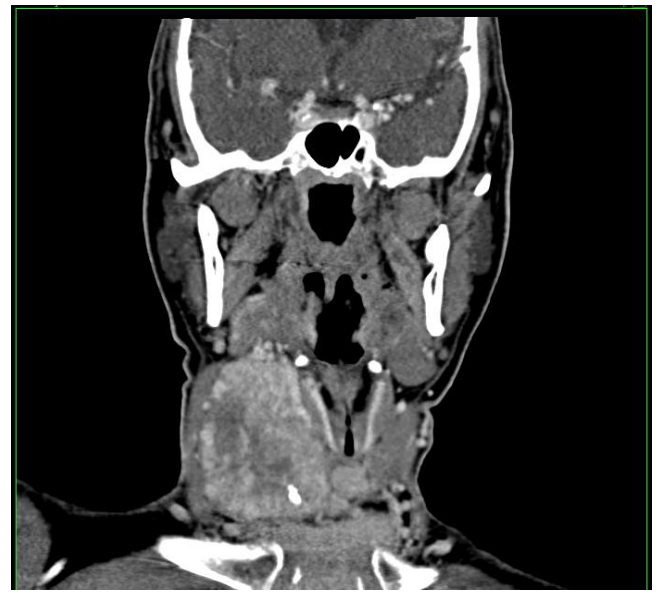

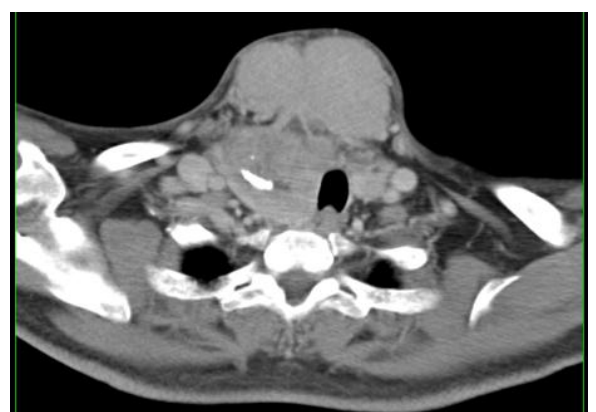

Fig 2

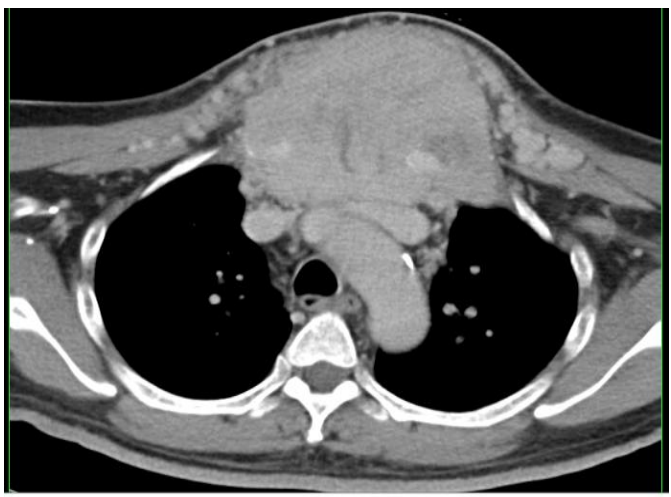

Fig 4

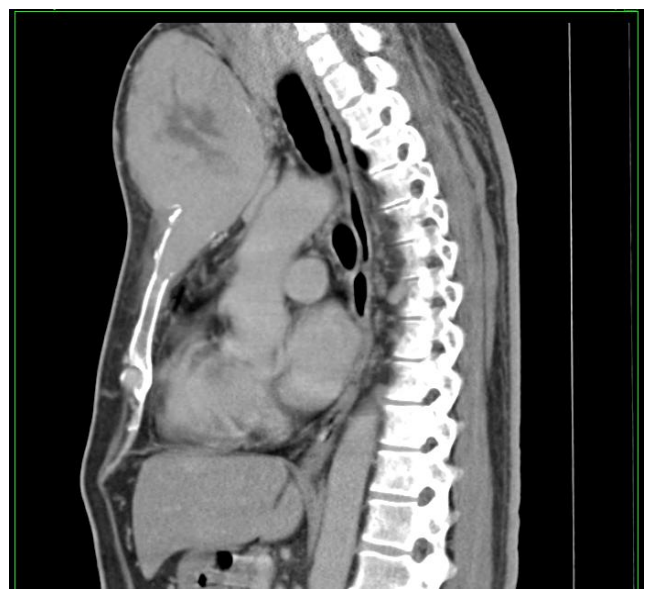

Fig $5 \quad$ Fig 6 
Figs 1-6: 1) Axial view of the CT showing a heterogenous Thyroid mass with malignant features. 2) Axial CT shows the mass has displaced the Thracea to the left but not infiltrating to major vessels of the neck. 3) Axial view of the CT showing the thyroid mass extending inferiorly and superficially at the upper thorax. 4) Axial view of the CT showing complete destruction of the sternum at the level of superior mediastinum and replaced by the tumour. 5) Coronal view of the neck CT shows the lesion is more prominent on the right Thyroid lobe. 6) Sagittal view of the neck CT shows the tumour destruction in relation to the sternum and compression to the mediastinal structures.

A few days later, he underwent a total thyroidectomy, sternectomy, rib resection and a fasciocutaneous free flap reconstruction harvested from Anterolateral Thigh Flap. Intraoperatively, it was found that the highly vascular tumor has invaded the sternum both anterior and posteriorly with encasement of the medial part of the clavicle, 1 st, 2nd and 3rd rib bilaterally. It also was adhered to the superior vena cava and innominate vein posteriorly. Multiple lung metastases felt throughout both lungs. No pleural or pericardial deposits seen. All the neurovascular structures identified, isolated and preserved. Fasciocutaneus flap measuring18 x $9 \mathrm{~cm}$ harvested from from right Anterolateral thigh to close chest wound defect of $17 \times 8 \mathrm{~cm}$ and chest fascial defect of $17 \times 15 \mathrm{~cm}$. The free flap donor artery was Descending Branch of Right Lateral Circumflex Femoral Artery which was anastomosed to Superior Thyroid Artery at recipient site. The donor vein which was the Vena Commitantes anastomosed to Superior Thyroid Vein at the recipent site. (Fig 7-10) 


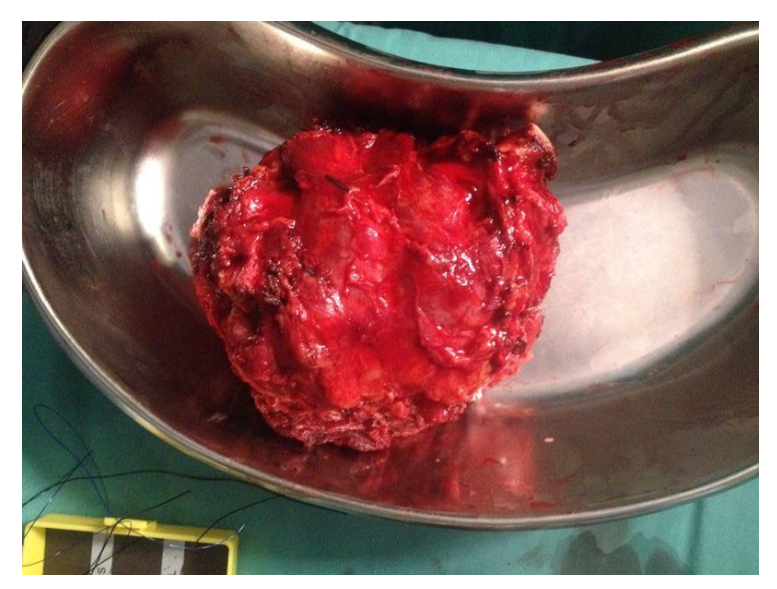

Fig 7

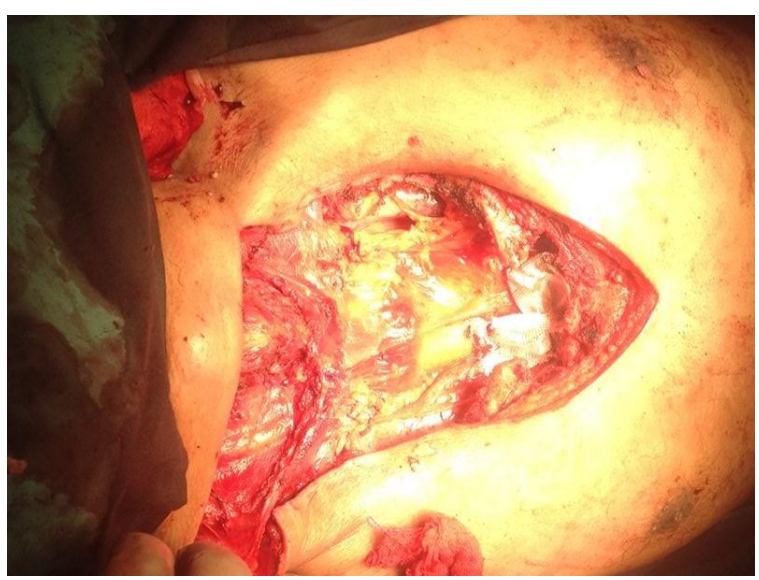

Fig 9

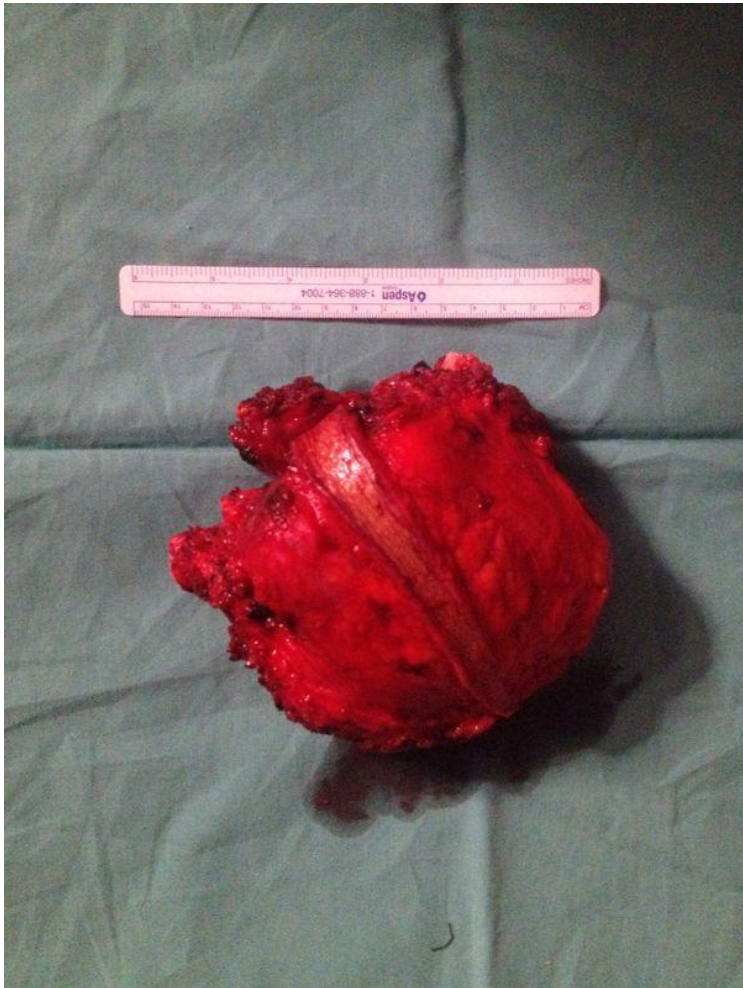

Fig 8

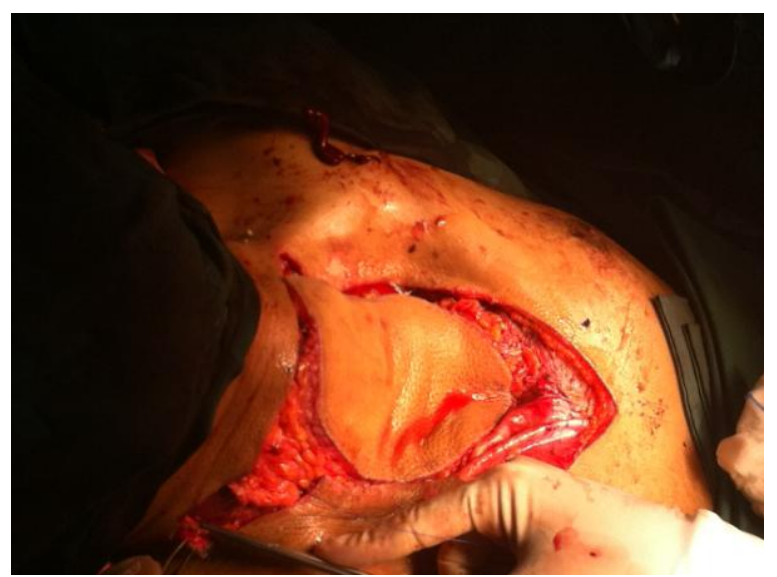

Fig 10

Figs 8-10: 7) The posterior view of the lesion showing sternum and resected end of clavicle. 8) The anterior view of the resected tumour with a strip of skin excised along with the lesion). 9) The wound defect left behind after the radical resection exposing the thyroid bed, pleura and superior mediastinum. 10) Fasciocutaneous flap placed in the wound defect and anastomosis reconstruction of donor artery to superior thyroid vessels. 
The surgery was uncomplicated and patient recovered fully post operation. However he had asymptomatic paradoxical movement of the flap on respiration at initial stage of recovery. Subsequently he underwent RAI ablation and was planned for a whole body scan later. We also started him on bilateral upper limb strengthening exercise and clavicle splint. Final HPE was reported as right thyroid lobe Follicular carcinoma with extra-thyroidal soft tissue margin is involved and left thyroid lobe local extension of Follicular carcinoma with free resection margin. Tumor extension to anterior wall chest confirmed of metastatic Follicular carcinoma.

\section{DISCUSSION}

Presentation with extracervical metastases occurs in up 5-10\% of advanced thyroid carcinoma. Patients frequently present with a chest mass and airway or esophagus compression symptoms. The most common cause of death in advanced thyroid carcinoma with extracervical metastases is asphyxia and this occurs in $26 \%$ of cases. Our focus in this case will be its extensive sternal and ribs invasion as this creates a huge dilemma in treatment options as there are only a few isolated cases reported in the literature of extensive sternal deposits in thyroid carcinoma. Surgery is still the primary treatment of choice in these cases and subsequently followed by radioiodine ablation with close monitoring of disease progression post-operatively.

In advanced metastatic thyroid carcinoma, surgery is often palliative and the basis for a surgical approach is achieving optimal hypotethical ablation due to reduced tumor load as a result of the surgery. In this case, tumor debulking could assist the patient in his further treatment and could give him a longer-than-expected life expectancy added on with a better cosmetic result. Ozaki et al in the 1990's has reported that reconstruction of the sternal region using acrylic resin and polypropylene mesh was feasible, however it has been proven to be 
unnecessary as reconstruction with polypropylene mesh was adequate. In our case, no reconstruction was attempted and a free myocutaneous flap was used to provide tissue cover for the patient post-operatively. This was due to the intra-operative en bloc nature of tumor resection with all first three ribs and clavicle resected would not allow mesh reconstruction. We also choose not to use acrylic because from our own experience in the past learned that acrylic caused problems with wound healing and especially in a patient whom to be rendered hypothyroid a month prior to RAI. A patient who is hypothyroid is more susceptible to severe infection at the acrylic reconstruction site.

As local tumor control was achieved post-operatively, it is now hoped that subsequent radioiodine ablation will improve the patient's disease-free period as suggested by Pak et al in 2003. They have suggested that metastasectomy will improve effectiveness of radioiodine administration post-operatively thus providing a discrete improvement in the patient's survival rate. This is especially true with adequate tumor resection in the presence of pulmonary deposits as observed by Kuauhyama Luna-Ortiz in 2012 whereby a reduction in tumor load assists in treatment of pulmonary deposits, which is not amenable by surgery. Hypothetical potentiation of radioiodine ablation also contributes to prevention of symptoms associated with these lesions such as pain, ulceration and dyspnea, all which occurs at a later stage and will definitely affect the patient's quality of life.

\section{CONCLUSION}

As a conclusion, Follicular thyroid carcinoma with bulky and extensive sternal extension requires significant palliation with surgery and subsequent radioiodine ablation. In the presence of pulmonary metastases, it allows the patient to have a near-normal quality of life. Careful planning of treatment options and a multidisciplinary approach enhances overall 
survival and concurrently quality of life. From surgeon's perspective, Superior Thyroid Artery and Vein should be considered as a potential anastomosis site if free flap tissue closure is required in debulking thyroidectomy. 


\section{REFERENCES}

1. Grubbs EG, Rich TA, Li G, Sturgis EM, Younes MN, Myers JN, et al. Recent advances in thyroid cancer. Curr Probl Surg 2008;45(3):156-250

2. Hundahl SA, Fleming ID, Fremgen AM, Menck HR. A National Cancer Data Base report on 53,856 cases of thyroid carcinoma treated in the U.S., 1985-1995 [see commetns]. Cancer 1998;83(12):2638-2648.

3. Harness JK, McLeod MK, Thompson NW, Noble WC, Burney RE. Deaths due to differentiated thyroid cancer: a 46-year perspective. World J Surg 1988;12(5):623-629.

4. Ozaki O, Kitagawa W, Koshiishi H, Sugino K, Mimura T, Ito K, et al. Thyroid carcinoma metastasized to the sternum: resection of the sternum and reconstruction with acrylic resin. J Surg Oncol 1995;60(4):282-285.

5. Pak H, Gourgiotis L, Chang WI, Guthrie LC, Skarulis MC, Reynolds JC, et al. Role of metastasectomy in the management of thyroid carcinoma: the NIH experience. $\mathrm{J}$ Surg Oncol 2003;82(1):10-18.

6. Kitamura Y, Shimizu K, Nagahama M, Sugino K, Ozaki O, Mimura T, et al. Immediate causes of death in thyroid carcinoma: clinicopathological analysis of 161 fatal cases. J Clin Endocrinol Metab 1999;84(11):4043-4049.

7. Eroglu A1, Karaoglanoglu N, Bilen H, Gursan N. Follicular thyroid carcinoma: metastasis to the sternum, 13 years after total thyroidectomy.

8. Waseem M Hajjar1, Sami Alnassar, Saleh N Abu-Daff, Saleh F Al-Dhahri. A novel technique for the reconstruction of resected sternoclavicular joints: A case report with a review of the literature. 